\title{
Intraspecific interactions shift from competitive to facilitative across a low to high disturbance gradient in a salt marsh
}

\author{
Liwen Zhang $\cdot$ Bingchen Wang
}

Received: 7 January 2016/Accepted: 24 May 2016/Published online: 1 June 2016

(C) Springer Science+Business Media Dordrecht 2016

\begin{abstract}
Disturbances are ubiquitous among salt marshes, and disturbances such as litter removal may form stressful environmental conditions. The stressgradient hypothesis $(\mathrm{SGH})$ predicts that the relative importance of facilitation and competition between species will vary inversely to each other across abiotic stress gradients. However, how intraspecific interactions vary across disturbance levels, and whether they follow the SGH has not been investigated. To test the SGH with intraspecific interactions within Suaeda salsa individuals, we assessed individual survival in established fully mapped salt marsh plots with two treatments, disturbed, litter removal and control, undisturbed plots. Recently developed spatial statistics were applied to distinguish between random processes, intraspecific facilitation, scramble competition, and contest competition underlying the spatial patterns at different spatial scales, growth stages, and disturbance levels. We found evidence that intraspecific interactions among $S$. salsa individuals across disturbance levels tended to support the SGH, but this
\end{abstract}

Communicated by Stephen Brewer.

L. Zhang $(\bowtie) \cdot$ B. Wang

Yantai Institute of Coastal Zone Research, Chinese

Academy of Sciences, Yantai 264003, China

e-mail: lwzhang@yic.ac.cn

B. Wang

University of Chinese Academy of Sciences,

Beijing 100049, China support depended on time and space. Intraspecific interactions were more likely to show positive density dependence in disturbed plots, particularly at the seedling period and when individuals were separated by $<9 \mathrm{~cm}$. However, positive density dependence was not detected in undisturbed plots. The protective effects of litter on seedlings, which were lost in undisturbed sites, may have been at least partly simulated at high seedling densities. Mortality in disturbed sites was not random, and facilitation had a more important influence than competition on population dynamics in high disturbance sites. However, the influence of competition on population dynamics, specifically scramble competition was also clearly important.

Keywords Intraspecific facilitation - Intraspecific competition - Salt marsh - Temporal scale $\cdot$ Spatial point pattern analysis $\cdot$ Disturbance

\section{Introduction}

Disturbances such as high tides, strong wave, strong wind, flooding, winter ice (high-latitude salt marsh), and human activities are ubiquitous in salt marshes (Ewanchuk and Bertness 2003; Tessier et al. 2002). One consequence of disturbance is litter removal. Litter (including in situ standing dead plant stems and scattered litter on the ground) appear to aid 
regeneration and establishment of seedlings in salt marshes by reducing water loss, salinity through shading, and keeping seeds and sheltering seedlings from cold winds in the winter and early spring. Thus, natural or anthropogenic removal of litter may influence species interactions and vegetation distribution. For example, litter are removed by high tides in winter in Yellow River Delta (personal observation) which turn the dense litter into thin ones even bare land. Litter removed by natural forces are deposited as mats. It has been suggested that these disturbances can facilitate species colonization, drive vegetation succession and species invasion, and improve species diversity through invasion of vacant patches by pioneer species (Minchinton 2002; Pennings and Richards 1998). However, the impact of in situ litter on species interactions is rarely evaluated. Holdredge and Bertness (2011) found litter to be crucial for the invasion of Phragmites, and prescribed litter removal to inhabit spread of Phragmites toward native species Juncus. Nevertheless, to our knowledge, the influence of litter on intraspecific interactions in salt marshes has not been studied.

The stress-gradient hypothesis (SGH) states that the frequency or importance of facilitative and competitive interactions between species will vary inversely to each other across abiotic or biotic stress gradients (Bertness and Callaway 1994). Evidence supporting this hypothesis in most of ecosystems with harsh environments is growing (He et al. 2013; Zhang et al. 2016). However, most tests of the SGH investigate interspecific interactions, with a few investigating the genetic consequence of the SGH (Castellanos et al. 2014; Castro et al. 2013; Zhang and Shao 2013). Intraspecific interactions may play a more important role than interspecific interactions in shaping populations and communities in harsh environments (GarciaCervigon et al. 2013; Martorell and Freckleton 2014). Testing the SGH at the single species level is a way to understand the influence of intraspecific facilitation and competition on population dynamics across stress gradients. Negative density dependence (e.g., selfthinning) is thought to be an important process influencing population and community dynamics, because intraspecific competition is traditionally thought to be stronger than interspecific interactions due to the greater niche overlap between conspecific individuals (Stoll and Prati 2001). However, stressful conditions may trigger a switch in overall intraspecific interactions from negative to positive interactions as proposed by the SGH (Chu et al. 2008; Fajardo and McIntire 2011; Sans et al. 2002). For example, Goldenheim et al. (2008) revealed that intraspecific interactions among individuals of Suaeda linearis showed positive density dependence in conditions with higher temperatures and greater evaporation stress, but exhibited negative density dependence in benign conditions, because the amelioration effect of neighbors outweighed the competition effect for resources under stressful conditions.

Species interactions across stress gradients depend on time and space (de la Cruz et al. 2008). Specifically, species interactions can vary between life-history stages. Studies have revealed species interactions to shift from facilitation to competition as seedlings grew into adult individuals (Callaway 1995; Callaway and Walker 1997; Goldenheim et al. 2008; Miriti 2006). The mechanism stems the vulnerable seedlings being sheltered by neighboring seedlings, alleviating stressful conditions. When seedlings grow up into adults, the ameliorative effects reduce and eventually competition for resource overrides facilitation by neighbors.

Spatial factors also influence species interactions because biotic processes such as scramble competition, contest competition, and facilitation may operate in different spatial scales (Das et al. 2008; Raventos et al. 2010). Scramble competition and contest competition are two contrary mechanisms of population density dependence. Scramble competition occurs when a limited resource is partitioned evenly among all individuals, causing dense clumps of individuals to die due to insufficient resources. Contest competition, on the other hand, occurs if the limited resource is partitioned unequally among individuals, resulting in death of some individuals due to resource insufficiencies, but survival of the competitors that acquire more resources (Raventos et al. 2010).

Spatial point pattern analysis is an effective tool to explore and separate spatial or biological processes underlying spatial patterns such as random process, facilitation, and scramble and contest competition at multiple spatial scales with observational data spanning long time periods. For example, scramble competition will lead to spatial segregation of surviving and dead individuals, while contest competition will cause spatial aggregation of surviving and dead individuals (Raventos et al. 2010). 
In this study, we investigated how the direction of intraspecific interactions among individuals of the species Suaeda salsa, common species and an annual herb in salt marsh of Yellow River Delta (China), change across different disturbance levels (litter removal and undisturbed control plots) at different spatial scales (from 0 to $15 \mathrm{~cm}$ ) and life stages (the seedling stage and the rapid growth stage). We predict that (1) according to the SGH, intraspecific interactions among $S$. salsa individuals will exhibit facilitation more frequently within litter removal plots compared to undisturbed control plots, (2) intraspecific interactions will occur at small spatial scales because of the small size of $S$. salsa individuals, and (3) scramble competition will be the major underlying processes driving $S$. salsa population dynamics under benign conditions, based on the results from previous studies (Raventos et al. 2010).

\section{Methods}

Study site

The study was conducted in the Yellow River Delta National Reserve, which is located in Shandong Province, northeast China $\left(37^{\circ} 40^{\prime} \mathrm{N}-38^{\circ} 10^{\prime} \mathrm{N}\right.$, $\left.118^{\circ} 41^{\prime} \mathrm{E}-119^{\circ} 16^{\prime} \mathrm{E}\right)$. The climate of this region is warm temperate, with an average annual temperature of $12.1^{\circ} \mathrm{C}$. Average annual precipitation is $551.6 \mathrm{~mm}$, falling mainly in the summer, and average annual evaporation is $1962 \mathrm{~mm}$. Tidal fluctuations in this region are irregularly semidiurnal. The dominant species within this salt marsh of this area were $S$. salsa, Phragmites australis, and Tamarix chinensis.

Experiment design, mapping, and individual monitoring

In order to evaluate the influences of disturbance (i.e., litter removal) on intraspecific interactions of dominant species $S$. salsa across growth stages, we established $180.5 \times 0.5 \mathrm{~m}$ plots in May 2013. We assigned a disturbance treatment to nine plots, where $S$. salsa litter was removed throughout each plot. The remaining nine plots were unaltered control treatments (Table 1). At the beginning of the study period, every individual within each plot was censured by
Table 1 Treatments

\begin{tabular}{llll}
\hline Plot & Treatments & Repeat & Disturbance \\
\hline L & Control & 9 & No \\
RL & Litter removal & 9 & Yes \\
\hline
\end{tabular}

recording species name and coordinates within the plot. Specifically, we fixed and marked one corner of the plot as the origin of coordinate axes, and we also fixed and marked the $x$-axis and $y$-axis. After that, we placed one ruler at each side of the plot. Then, we tagged each individual with a plastic ring and there was a unique number on the ring. Finally, we recorded the coordinates of each individual according to the scales of the rulers. Plots were re-censored in June and September 2013. As there were few individuals of species other than $S$. salsa, we collected data only on S. salsa.

To assess the effect of litter removal on soil salinity, we measured electronic conductivity (EC) of soil in each plot at May, late June, and September with in situ electronic conductivity meter. The changes of EC from May, late June to September in the same plot were calculated as follows: "EC at September-EC at late June" and "EC at late June-EC at May." Then, we compared the change of soil salinity between litter removal plots and control plots with one-way ANOVA.

Spatial pattern analysis

We performed scale-dependent point pattern analysis to analyze qualitatively marked point patterns of $S$. salsa in replicated plots, i.e., each plant at a given growth stage can have the marks "dead" or "alive," and subscript 1 indicates dead individuals while subscript 2 indicates alive individual in the following formulas of test statistics. Three-test statistics, a univariate $g_{11}(r)$ function, a bivariate $g_{12}(r)$ function, and a bivariate difference $g_{1,1+2}-g_{2,1+2}(r)$ function were employed to describe spatial patterns (Jacquemyn et al. 2010; Wiegand and Moloney 2004). $\hat{g}_{12}(r)$ is an estimator of the bivariate pair-correlation function $g_{12}(r)$, which is calculated based on the $O$-ring statistic $O_{12}(r)$ (Wiegand and Moloney 2004). Calculations of $\hat{O}_{12}^{\mathrm{w}}(r)$ [an estimator of $O_{12}(r)$ ] and $\hat{g}_{12}(r)$ are as follows: 
$\hat{O}_{12}^{\mathrm{w}}(r)=\frac{\frac{1}{n_{1}} \sum_{i=1}^{n_{1}} \operatorname{Points}_{2}\left[R_{1, i}^{\mathrm{w}}(r)\right]}{\frac{1}{n_{1}} \sum_{i=1}^{n_{1}} \operatorname{Area}\left[R_{1, i}^{\mathrm{w}}(r)\right]}$

$\hat{g}_{12}(r)=\frac{\hat{O}_{12}^{\mathrm{w}}(r)}{\hat{\lambda}_{2}}$,

where $r$ is the radius of the sampling $\operatorname{ring} R_{1, i}^{\mathrm{w}}(r) ; w$ is the width of the ring; and the ring is centered around the $i$ th individual within pattern 1 (dead plants); Points $_{2}\left[R_{1, i}^{\mathrm{w}}(r)\right]$ refers to the number of individuals making up pattern 2 (alive plants) of sampling ring $R_{1, i}^{\mathrm{W}}(r)$, and $\operatorname{Area}\left[R_{1, i}^{\mathrm{W}}(r)\right]$ denotes the area of this sampling ring; $n_{1}$ is the number of individuals within pattern $1 ; \lambda_{2}$ is the intensity of individuals within pattern 2 and $\hat{\lambda}_{2}$ is an estimator of $\lambda_{2}$. The univariate $g_{11}(r)$ function is used to reveal aggregation patterns of dead plants. The bivariate pair-correlation function $g_{12}(r)$ quantifies correlations such as attraction or segregation between dead and surviving individuals, and the $g_{1,1+2}-g_{2,1+2}(r)$ function reveals densitydependent mortality patterns.

Random labeling analysis was performed to structure the null model, and detect departures of observed spatial patterns from the null model, which point to non-random impacts of intraspecific interactions on plant mortality. The random labeling null model was constructed by randomly assigning a location and status (alive or dead) to each observed individual (including surviving and dead individuals), then calculating statistics, $g_{11}(r), g_{12}(r), g_{1,1+2}-g_{2,1+2}(r)$, using the simulated data. The null model was simulated 999 times and the simulation results plus the observed patterns formed the simulation envelopes (Baddeley et al. 2014).

The ecological interpretations of departures of the univariate and bivariate pair-correlation functions from the null model are as follows: (1) The $g_{11}(r)$ function reveals clustering of dead individuals, and $g_{11}(r)$ values exceeding the simulation envelopes indicate aggregation of dead individuals; (2) the $g_{12}(r)$ function reveals attraction or segregation of dead and surviving plants, with $g_{12}(r)$ values lower than the simulation envelopes suggesting separation of dead and surviving individuals, and therefore, scramble competition. Otherwise, $g_{12}(r)$ values exceeding the simulation envelopes point toward contest competition; (3) the $g_{1,1+2}-g_{2,1+2}(r)$ function detects density-dependent mortality. Values for $g_{1,1+2}-g_{2,1+-}$ ${ }_{2}(r)$ that are lower than the simulation envelopes point toward positive density dependence of deaths of individuals. That is, there are positive interactions between surviving individuals. Otherwise, $g_{1,1+2}$ $g_{2,1+2}(r)$ values above the simulation envelopes indicate that deaths of individuals are caused by negative density dependence; (4) values for $g_{11}(r)$, $g_{12}(r), g_{1,1+2}-g_{2,1+2}(r)$ falling within the simulation envelopes are suggestive of random patterns in plant deaths with no obvious effect of species competition or facilitation.

A goodness-of-fit test was conducted to test the significance of intraspecific interactions without taking account of spatial scales. This test was a complement to analyses based on simulation envelopes. All spatial analyses were conducted with the Programita software (Wiegand and Moloney 2004).

\section{Results}

From Fig. 1, we found that the differences of change of soil salinity in the same plot between litter removal plots and control plots during the experiment related to seasons. The difference from May to late June was non-significant. However, the difference from late June to September was significant. The soil salinity increased in the same plot for litter removal plots from late June to September, while the soil salinity decreased in the same plot for control plots from late June to September.

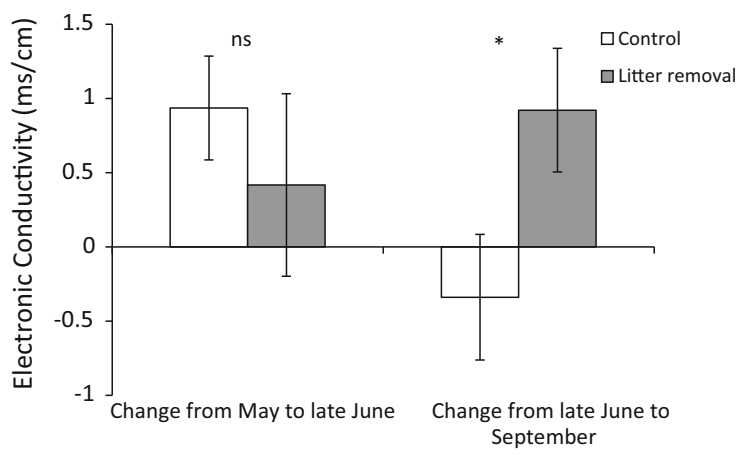

Fig. 1 The change of soil salinity measured as the electronic conductivity in the same plot during the experimental periods (May to late June, late June to September) for control plots and litter removal plots. The error bar is $\pm \mathrm{SE}, n=9$. Asterisk indicates $P$ value $<0.05$, and "ns" means non-significance 
Table 2 Statistic significance of pair-correlation functions in different treatments and growth stages obtained from a goodness-of-fit test

\begin{tabular}{llll}
\hline & Function & $p$ value & Sig. \\
\hline L6-5 & $g_{11}$ & 0.039 & $*$ \\
& $g_{12}$ & 0.021 & $*$ \\
& $g_{1,1+2}-g_{2,1+2}$ & 0.726 & $\mathrm{~ns}$ \\
RL6-5 & $g_{11}$ & 0.528 & $\mathrm{~ns}$ \\
& $g_{12}$ & 0.001 & $* *$ \\
& $g_{1,1+2}-g_{2,1+2}$ & 0.003 & $* *$ \\
L9-6 & $g_{11}$ & 0.046 & $*$ \\
& $g_{12}$ & 0.055 & $\mathrm{~ns}$ \\
& $g_{1,1+2}-g_{2,1+2}$ & 0.849 & $\mathrm{~ns}$ \\
RL9-6 & $g_{11}$ & 0.363 & $\mathrm{~ns}$ \\
& $g_{12}$ & 0.073 & $\mathrm{~ns}$ \\
& $g_{1,1+2}-g_{2,1+2}$ & 0.148 & $\mathrm{~ns}$ \\
\hline
\end{tabular}

$n s$ non-significance

$0.01<* p<0.05 ; * * p<0.01$

From the goodness-of-fit stats (Table 2), we found that $g_{11}(r)$ function values of L6-5 and L9-6 indicated significant aggregation of plant mortalities. The $g_{12}(r)$ function values of L6-5 and RL6-5 suggested significant scramble competition, and the $g_{1,1+2^{-}}$ $g_{2,1+2}(r)$ value of RL6-5 showed significantly positive interactions among individuals.

Figure 2 shows $S$. salsa results of spatial point pattern in different treatment plots across seasons at specific spatial scales. Results, in the order of growth stages of $S$. salsa, are as follows. Specifically, spatial scale 0 indicates that spatial distances range from 0 to $1 \mathrm{~cm}$; spatial scale 1 means that spatial distances range from 1 to $2 \mathrm{~cm}$; spatial scale 2 indicates that spatial distances range from 2 to $3 \mathrm{~cm}$; and so forth.

1. From early May to late June, which was the seedling period of $S$. salsa, the spatial structure function $g_{11}(r)$ of control plot L6-5 revealed that at spatial distances 1-2 cm, the death of individuals were aggregated, while mortality of individuals at other spatial distances were random (Fig. 2a). The $g_{12}(r)$ function of control plot L65 at distances $0-2 \mathrm{~cm}$ was lower than the simulation envelopes, suggesting scramble competition among $S$. salsa individuals (Fig. 2b). The $g_{1,1+2}-g_{2,1+2}(r)$ function of control plot L6-5 detected species facilitation at spatial distances of
$0-1 \mathrm{~cm}$ (Fig. 2c). The $g_{11}(r)$ of litter removal plot RL6-5 showed that mortalities were randomly distributed with spatial distances of $0-16 \mathrm{~cm}$ (Fig. 2d). The $g_{12}(r)$ values of litter removal plot RL6-5 were lower than the simulation envelopes at spatial distances of $1-6$ and $7-8 \mathrm{~cm}$, which suggested scramble competition among individuals (Fig. 2e). The $g_{1,1+2}-g_{2,1+2}(r)$ function of litter removal plot RL6-5 was lower than the simulation envelopes at spatial distances of $2-3$ and $4-5 \mathrm{~cm}$, which suggested intraspecific facilitation at these spatial scales (Fig. 2f).

2. From late June to September, which was the rapid growth period of $S$. salsa, $g_{11}(r), g_{12}(r), g_{1,1+2^{-}}$ $g_{2,1+2}(r)$ values of control plots L9-6 fell within the simulation envelope at all spatial scales $(0-15 \mathrm{~cm})$, suggesting the spatial patterns in mortalities were random (Fig. $2 \mathrm{~g}-\mathrm{i}$ ). The $g_{11}(r)$ values of litter removal plot RL9-6 exhibited random spatial distribution (Fig. 2j), while $g_{12}(r)$ of litter removal plot RL9-6 revealed scramble competition at the spatial scales of $0,2,4,12$, and 14 (Fig. $2 \mathrm{k}$ ). The $g_{1,1+2}-g_{2,1+2}(r)$ values of litter removal plot RL9-6 revealed positive density dependence at the spatial scale of $8 \mathrm{~cm}$ (Fig. 2l).

\section{Discussion}

We used recently developed "individual-based" spatial statistics to analyze spatial patterns in mortalities among conspecific individuals across disturbance levels and growth stages. This technique is often used in analyses of species interactions among long-lived plants, such as trees or shrubs, in fully mapped plots (Pillay and Ward 2012; Queenborough et al. 2007; Raventos et al. 2010; Yu et al. 2009). In our study, this technique was applied to study interactions among individuals of an annual herb species, taking advantage of reductions in required disturbances of the plant community during the experiment. This technique also improved detection of spatiotemporal changes of species interactions achieved with "individual-based" spatial statistics.

Comparing intraspecific interactions of $S$. salsa between litter removal and control plots, we found that intraspecific interactions within litter removal plots were more likely to show positive density dependence 

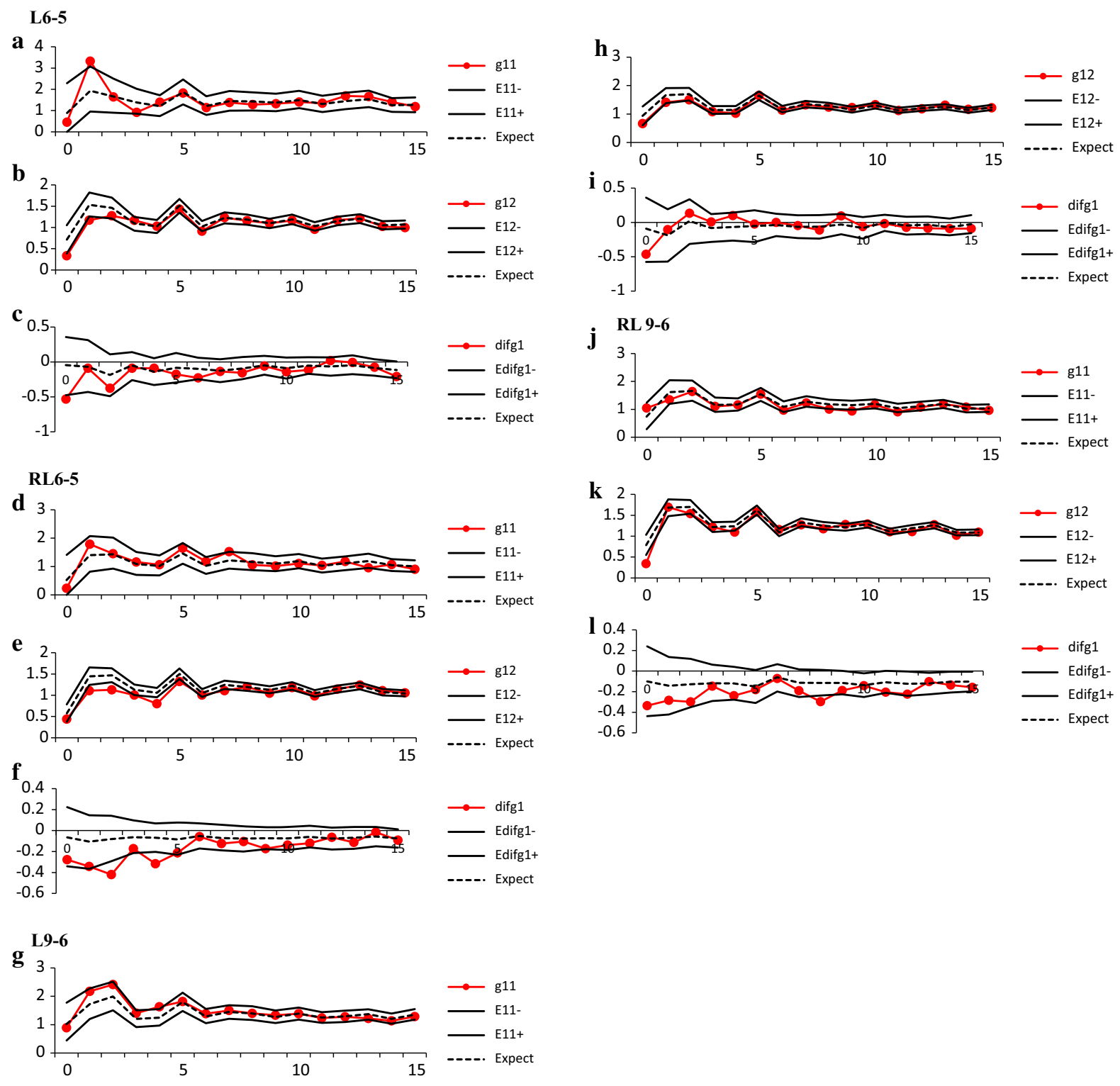

during the seedling period. In control plots, on the other hand, positive density dependence was not detected.

Although we found evidence of an intraspecific facilitative effect following litter removal early in the growing season, the mechanism of positive density dependence remains uncertain and does not appear to be related to a reduction in soil salinity. Litter removal did not increase soil salinity levels early in the growing season (from May to June). We nonetheless suggest that the presence of litter and/or conspecific neighbors

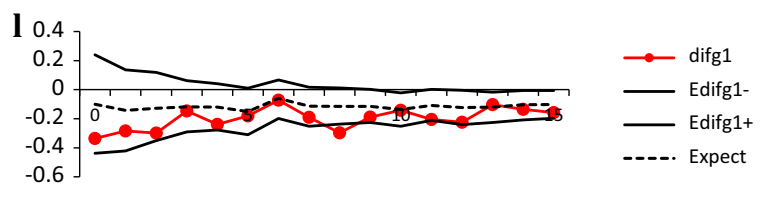

protected seedlings from damaging or desiccating strong winds, which were much colder and stronger in the spring than later in the growing season (personal observations). We hypothesize that when litter was experimentally removed, conspecific neighbors played a more important role in protecting seedlings from damaging spring winds. If our hypothesis is correct, then our results are consistent with the SGH, which predicts that facilitation should be relatively more important than competition when abiotic stress increases (Lortie and Callaway 2006). 
4Fig. 2 Intraspecific interactions of Suaeda salsa varying with disturbance levels, spatial distances, and seasons. L6-5 a-c is the variation of intraspecific interactions of $S$. salsa along spatial scales $(0-15 \mathrm{~cm})$ in control plots during May to late June 2013; L9-6 $\mathbf{g}-\mathbf{i}$ is the variation of intraspecific interactions of $S$. salsa with spatial scales $(0-15 \mathrm{~cm})$ in control plots during late June to September 2013; RL6-5 d-f is change of intraspecific interactions of $S$. salsa with spatial scales $(0-15 \mathrm{~cm})$ in disturbance treatment plots during May to late June 2013; RL9-6 j-I is change of intraspecific interactions of $S$. sals $a$ with spatial scales $(0-15 \mathrm{~cm})$ in disturbance treatment plots during late June to September 2013. The $g_{11}$ (L6-5 a, RL6-5 d, L9-6 g, RL9-6 j), $g_{12}$ (L6-5 b, RL6-5 e, L9-6 h, RL9-6 k), $g_{1,1+2}-g_{2,1+2}$ (i.e., "diffg1" in L6-5 c, RL6-5 f, L9-6 i, RL9-6 l) functions were used to evaluate spatial patterns of dead ("1" denoted dead individuals) and surviving individuals (" 2 " indicated surviving individuals). The empirical values of the above spatial pattern functions are shown as a solid line with solid circles. Black solid lines ("E11-," "E11+," "E12-," "E12+," "Ediffg1-," "Ediffg1+") are the $95 \%$ envelopes of values from 1000 random simulations for the null model. The dashed "Expect" line is the expectation of the null model. The $x$-axis is the spatial scale $(\mathrm{cm})$. The $g_{11}(r)$ function reveals clustering of dead individuals, and $g_{11}(r)$ values exceeding the simulation envelopes indicate aggregation of dead individuals. The $g_{12}(r)$ function detects scramble and contest competition, with $g_{12}(r)$ values lower than the simulation envelopes suggesting scramble competition; otherwise, $g_{12}(r)$ values exceeding the simulation envelopes point toward contest competition. The $g_{1,1+2}-g_{2,1+2}(r)$ function detects density-dependent mortality. Values for $g_{1,1+2}-g_{2,1+2}(r)$ that are lower than the simulation envelopes point toward positive interactions between surviving individuals; otherwise, $g_{1,1+2}-g_{2,1+2}(r)$ values above the simulation envelopes indicate negative density dependence. Values for $g_{11}(r), g_{12}(r), g_{1,1+2}-g_{2,1+2}(r)$ falling within the simulation envelopes are suggestive of random patterns in plant deaths with no obvious effect of species competition or facilitation. L6-5. a $g_{11}(r)$ in control plots from May to late June (detecting clustering of dead individuals). b $g_{12}(r)$ in control plots from May to late June (detecting scramble vs. contest competition). c $g_{1,1+2}(r)-g_{2,1+2}(r)$ in control plots from May to late June (detecting positive vs. negative density dependence). RL6-5. d $g_{11}(r)$ in litter removal plots from May to late June (detecting clustering of dead individuals). e $g_{12}(r)$ in litter removal plots from May to late June (detecting scramble vs. contest competition). f $g_{1,1+2}(r)-g_{2,1+2}(r)$ in litter removal plots from May to late June (detecting positive vs. negative density dependence). L9-6 $\mathbf{g} g_{11}(r)$ in control plots from June to September (detecting clustering of dead individuals). $\mathbf{h} g_{12}(r)$ in control plots from June to September (detecting scramble vs. contest competition). $\mathbf{i} g_{1,1+2}(r)-g_{2,1+2}(r)$ in control plots from June to September (detecting positive vs. negative density dependence). RL 9-6 j $g_{11}(r)$ in litter removal plots from June to September (detecting clustering of dead individuals). $\mathbf{k} g_{12}(r)$ in litter removal plots from June to September (detecting scramble vs. contest competition). $\mathbf{l} g_{1,1+2}(r)-g_{2,1+2}(r)$ in litter removal plots from June to September (detecting positive vs. negative density dependence)
The positive density dependence of $S$. salsa interactions varied temporally, with positive density dependence more likely to occur during the seedling establishment period. This result is consistent with previous studies (Callaway and Walker 1997; Jensen et al. 2012; Lortie and Turkington 2008). Thus, we confirm that population dynamics are highly influenced by positive density dependences, especially for the recruitment and establishment stages when individuals encounter disturbance. We also have shown that litter play a key role in intraspecific interactions, and therefore, population dynamics in the salt marsh.

Ecologists have argued for the importance of incorporation of spatial scales into the study of ecological processes which underlie spatial patterns because different biological processes may operate at distinguishing spatial scales (Borcard and Legendre 2002; Chase and Leibold 2002; Wiegand et al. 2007). Our results support this argument. Spatial distance among individuals was a very important factor influencing facilitation among $S$. salsa individuals. For example, intraspecific facilitation occurred among individuals, which were separated by less than $9 \mathrm{~cm}$ (Fig. 2) and scramble competition among individuals seemed to occur when individuals were separated by $6 \mathrm{~cm}$ (Fig. 2).

Although intraspecific facilitation was more important than intraspecific competition at high disturbance levels (i.e., litter removal plots), intraspecific competition still played an essential role in population dynamics. We found that regardless of disturbance levels (litter removal or control plots), intraspecific interactions among $S$. salsa individuals during the seedling period exhibited scramble competition, but there was no sign of scramble competition or contest competition during the rapid growth period. This finding suggests that segregation of surviving and dead individuals observed in this study was caused by scramble competition for limited resources. With the limited resources within the study plots being equally partitioned among competitors, dense clusters of $S$. salsa individuals could not acquire sufficient resources to survive, leading to aggregation mortalities. Although plant mortality was random at many spatial scales, observations of density-dependent mortalities among S. salsa within high-stress salt marsh plots, 
suggest that overall, plant mortality was not random; i.e., death was not equally probable for all individuals within a community (Getzin et al. 2006).

In summary, intraspecific interactions among closely neighboring $S$. salsa individuals exhibited positive density dependence in disturbance treatment plots, where litter were removed during the seedling period, but not in control plots. This result seemed to support the SGH. The random mortality hypothesis, on the other hand was rejected due to non-random mortalities among individuals. Evidence of nonrandom mortalities was seen in positive density dependence of mortalities at high disturbance levels and detection of scramble competition. Plant mortality appeared to have depended on the quantity of available limited resources, partitioning of the resources among individuals, and the density of individuals.

Acknowledgments This research is funded by National Natural Science Foundation of China (No. 31100313), the Foundation for Outstanding Young Scientist in Shandong Province (No. BS2013HZ012), and the Program of Science and Technology Service Network Initiative, Chinese Academy of Sciences (No. KFJ-EW-STS-127). We thank the reviewers and editor for their comments and suggestions, which have been very helpful for improving the manuscript. We would also like to thank Christine Verhille at the University of British Columbia for her assistance with English language and grammatical editing of the manuscript.

\section{References}

Baddeley A, Diggle PJ, Hardegen A, Lawrence T, Milne RK, Nair G (2014) On tests of spatial pattern based on simulation envelopes. Ecol Monogr 84:477-489

Bertness MD, Callaway R (1994) Positive interactions in communities. Trends Ecol Evol 9:191-193

Borcard D, Legendre P (2002) All-scale spatial analysis of ecological data by means of principal coordinates of neighbour matrices. Ecol Model 153:51-68

Callaway RM (1995) Positive interactions among plants. Bot Rev 61:306-349

Callaway RM, Walker LR (1997) Competition and facilitation: a synthetic approach to interactions in plant communities. Ecology 78:1958-1965

Castellanos MC, Donat-Caerols S, Gonzalez-Martinez SC, Verdu M (2014) Can facilitation influence the spatial genetics of the beneficiary plant population? J Ecol 102:1214-1221

Castro BM, Moriuchi KS, Friesen ML, Badri M, Nuzhdin SV, Strauss SY, Cook DR, von Wettberg E (2013) Parental environments and interactions with conspecifics alter salinity tolerance of offspring in the annual Medicago truncatula. J Ecol 101:1281-1287
Chase JM, Leibold MA (2002) Spatial scale dictates the productivity-biodiversity relationship. Nature 416:427-430

Chu CJ, Maestre FT, Xiao S, Weiner J, Wang YS, Duan ZH, Wang G (2008) Balance between facilitation and resource competition determines biomass-density relationships in plant populations. Ecol Lett 11:1189-1197

Das A, Battles J, van Mantgem PJ, Stephenson NL (2008) Spatial elements of mortality risk in old-growth forests. Ecology 89:1744-1756

de la Cruz M, Romao RL, Escudero A, Maestre FT (2008) Where do seedlings go? A spatio-temporal analysis of seedling mortality in a semi-arid gypsophyte. Ecography 31:720-730

Ewanchuk PJ, Bertness MD (2003) Recovery of a northern New England salt marsh plant community from winter icing. Oecologia 136:616-626

Fajardo A, McIntire EJB (2011) Under strong niche overlap conspecifics do not compete but help each other to survive: facilitation at the intraspecific level. $\mathrm{J}$ Ecol 99:642-650

Garcia-Cervigon AI, Gazol A, Sanz V, Camarero JJ, Olano JM (2013) Intraspecific competition replaces interspecific facilitation as abiotic stress decreases: the shifting nature of plant-plant interactions. Perspect Plant Ecol Evol Syst 15:226-236

Getzin S, Dean C, He FL, Trofymow JA, Wiegand K, Wiegand $\mathrm{T}$ (2006) Spatial patterns and competition of tree species in a Douglas-fir chronosequence on Vancouver Island. Ecography 29:671-682

Goldenheim W, Irving A, Bertness M (2008) Switching from negative to positive density-dependence among populations of a cobble beach plant. Oecologia 158:473-483

He Q, Bertness MD, Altieri AH (2013) Global shifts towards positive species interactions with increasing environmental stress. Ecol Lett 16:695-706

Holdredge C, Bertness MD (2011) Litter legacy increases the competitive advantage of invasive Phragmites australis in New England wetlands. Biol Invasions 13:423-433

Jacquemyn H, Endels P, Honnay O, Wiegand T (2010) Evaluating management interventions in small populations of a perennial herb Primula vulgaris using spatio-temporal analyses of point patterns. J Appl Ecol 47:431-440

Jensen AM, Lof M, Witzell J (2012) Effects of competition and indirect facilitation by shrubs on Quercus robur saplings. Plant Ecol 213:535-543

Lortie CJ, Callaway RM (2006) Re-analysis of meta-analysis: support for the stress-gradient hypothesis. J Ecol 94:7-16

Lortie CJ, Turkington R (2008) Species-specific positive effects in an annual plant community. Oikos 117:1511-1521

Martorell C, Freckleton RP (2014) Testing the roles of competition, facilitation and stochasticity on community structure in a species-rich assemblage. J Ecol 102:74-85

Minchinton TE (2002) Disturbance by wrack facilitates spread of Phragmites australis in a coastal marsh. J Exp Mar Biol Ecol 281:89-107

Miriti MN (2006) Ontogenetic shift from facilitation to competition in a desert shrub. J Ecol 94:973-979

Pennings SC, Richards CL (1998) Effects of wrack burial in saltstressed habitats: Batis maritima in a southwest Atlantic salt marsh. Ecography 21:630-638 
Pillay T, Ward D (2012) Spatial pattern analysis and competition between Acacia karroo trees in humid savannas. Plant Ecol 213:1609-1619

Queenborough SA, Burslem DFRP, Garwood NC, Valencia R (2007) Neighborhood and community interactions determine the spatial pattern of tropical tree seedling survival. Ecology 88:2248-2258

Raventos J, Wiegand T, De Luis M (2010) Evidence for the spatial segregation hypothesis: a test with nine-year survivorship data in a Mediterranean shrubland. Ecology 91:2110-2120

Sans FX, Escarre J, Lepart J, Hopkins F (2002) Positive vs. negative interactions in Picris hieracioides L., a mid-successional species of Mediterranean secondary succession. Plant Ecol 162:109-122

Stoll P, Prati D (2001) Intraspecific aggregation alters competitive interactions in experimental plant communities. Ecology 82:319-327

Tessier M, Gloaguen JC, Bouchard V (2002) The role of spatio-temporal heterogeneity in the establishment and maintenance of Suaeda maritima in salt marshes. J Veg Sci 13:115-122

Wiegand T, Moloney KA (2004) Rings, circles, and nullmodels for point pattern analysis in ecology. Oikos 104:209-229

Wiegand T, Gunatilleke S, Gunatilleke N, Okuda T (2007) Analyzing the spatial structure of a Sri Lankan tree species with multiple scales of clustering. Ecology 88:3088-3102

Yu H, Wiegand T, Yang XH, Ci LJ (2009) The impact of fire and density-dependent mortality on the spatial patterns of a pine forest in the Hulun Buir sandland, Inner Mongolia, China. For Ecol Manag 257:2098-2107

Zhang LW, Shao HB (2013) Direct plant-plant facilitation in coastal wetlands: a review. Estuar Coast Shelf Sci 119:1-6

Zhang L, Mi X, Shao H (2016) Phylogenetic relatedness influences plant interspecific interactions across stress levels in coastal ecosystems: a meta-analysis. Estuar Coasts. doi:10. 1007/s12237-016-0104-2 